\title{
Big-Data Visualization
}

\author{
Daniel Keim - University of Konstanz \\ Huamin Qu • Hong Kong University of Science and Technology
}

Kwan-Liu Ma - University of California, Davis

W e've entered a data-driven era, in which data are continuously acquired for a variety of purposes. The ability to make timely decisions based on available data is crucial to business success, clinical treatments, cyber and national security, and disaster management. Additionally, the data generated from large-scale simulations, astronomical observatories, high-throughput experiments, or high-resolution sensors will help lead to new discoveries if scientists have adequate tools to extract knowledge from them.

However, most data have become simply too large and often have too short a lifespan. Almost all fields of study and practice eventually will confront this big-data problem. Government agencies and large corporations are launching research programs to address big data's challenges. Visualization has proven effective for not only presenting essential information in vast amounts of data but also driving complex analyses. Big-data analytics and discovery present new research opportunities to the computer graphics and visualization community. This special issue highlights the latest advancements in solving the big-data problem via visual means, with four articles on new techniques, systems, or applications.

In "Customizing Computational Methods for Visual Analytics with Big Data," Jaegul Choo and Haesun Park discuss the interplay between precision and convergence-two aspects that haven't received appropriate consideration in visual analyses so far. The authors propose customizing computational methods to include low-precision computation and iteration-level visualizations to ensure real-time visual analytics for big data.

In "Feature Tracking and Visualization of the Madden-Julian Oscillation in Climate Simulation," Teng-Yok Lee and his colleagues present an integrated analysis and visualization framework for scientists to better understand the MaddenJulian oscillation (MJO) phenomenon from largescale spatiotemporal climate simulation data. The authors demonstrate how the tight integration of MJO domain knowledge, data analysis techniques such as feature tracking, and visualization methods such as Hovmöller diagrams and a virtual globe can lead to a powerful system for climate research.

In "Visualizing Large, Heterogeneous Data in Hybrid-Reality Environments," Khairi Reda and his colleagues show how a new kind of visualization space called hybrid-reality environments can achieve scalable visualization of heterogeneous datasets. These environments synergize the capabilities of VR and high-resolution tiled LCD walls, letting users juxtapose 2D and 3D datasets and create hybrid 2D$3 \mathrm{D}$ information spaces. The authors introduce two such environments-Cyber-Commons and CAVE2and some real-world applications.

Finally, in "Exploring the Connectome: Petascale Volume Visualization of Microscopy Data Streams," Johanna Beyer and her colleagues describe a system for interactive exploration of petascale volume data of neural tissues generated by high-throughput electron microscopy imaging. This visualization-driven system lets users handle multiple volumes and incomplete data, restricts most computations to a small subset of the data, and achieves scalable computing with a multiresolution virtual memory. The authors applied the system to a mouse cortex volume with a resolution of $21,494 \times 25,790 \times 1,850$ voxels.

W: e hope you enjoy these articles, which provide a very small sample of big-data visualization problems and solutions. We anticipate that 
the emerging big-data applications will present new challenges, drawing new interest and research that leads to many innovations and advancements.

We thank all the editors, authors, and reviewers who helped make this special issue a success. Those of you who wish to learn more will find a number of workshops, conferences, and committee reports devoted to big data. Several of these meetings feature sessions on visualization and analytics research. Examples include the First Workshop on Big-Data Visualization with the 2013 IEEE Conference on Big Data (http://vis.ucdavis.edu/ Workshops/BigDataVis2013), the IEEE Symposium on Large-Scale Data Analysis and Visualization with IEEE VIS 2013 (www.ldav.org), and the Ultrascale Visualization Workshop with Supercomputing 2013 (http://vis.cs.ucdavis.edu/Ultravis13).

Daniel Keim is a full professor and the head of the Information Visualization and Data Analysis Research Group in the University of Konstanz's Computer Science Department. Keim received a habilitation in computer science from the University of Munich. He has been program cochair of the IEEE Information Visualization Conference, the IEEE Conference on Visual Analytics Science and Technology (VAST), and the ACM SIGKDD Conference on Knowledge Discovery and Data Mining. He's on the steering committees of IEEE
VAST and the Eurographics Conference on Visualization. Contact him at keim@uni-konstanz.de.

Huamin Qu is an associate professor in the Hong Kong University of Science and Technology's Department of Computer Science and Engineering. His main research interests are visualization and computer graphics. Qu received a $P h D$ in computer science from Stony Brook University. He's on the steering committee of the IEEE Pacific Visualization Symposium and was the program cochair for the 2011 and 2012 symposiums. He also was the conference cochair for the International Symposium on Visual Information Communication and Interaction in 2011 and 2012. He received an Honorable Mention for the Best Paper Award at IEEE VIS 2009 and the 2009 IBM Faculty Award. Contact him at huamin@cse.ust.hk.

Kwan-Liu Ma is a professor of computer science at the University of California, Davis, where he leads the Visualization \& Interface Design Innovation research group and directs the UC Davis Center for Visualization. His research interests include visualization, high-performance computing, and interactive $3 D$ graphics. Ma received a $P h D$ in computer science from the University of Utah. He was a founding member of the IEEE Symposium on Large-Scale Data Analysis and Visualization. He's an IEEE Fellow and a member of the IEEE Computer Graphics and Applications editorial board. Contact him at ma@cs.ucdavis.edu. 